\title{
Exploring Sex and Gender Differences in Sleep Health: A Society for Women's Health Research Report
}

\author{
Monica P. Mallampalli, PhD, MS, and Christine L. Carter, PhD, MPH
}

\begin{abstract}
Previous attempts have been made to address sleep disorders in women; however, significant knowledge gaps in research and a lack of awareness among the research community continue to exist. There is a great need for scientists and clinicians to consider sex and gender differences in their sleep research to account for the unique biology of women. To understand the role of sex differences in sleep and the state of women's sleep health research, the Society for Women's Health Research convened an interdisciplinary expert panel of wellestablished sleep researchers and clinicians for a roundtable meeting. Focused discussions on basic and clinical research along with a focus on specific challenges facing women with sleep-related problems and effective therapies led to the identification of knowledge gaps and the development of research-related recommendations. Additionally, sex differences in sleep disorders were noted and discussed in the context of underlying hormonal differences. Differences in sleep behavior and sleep disorders may not only be driven by biological factors but also by gender differences in the way women and men report symptoms. Progress has been made in identifying sex and gender differences in many areas of sleep, but major research gaps in the areas of epidemiology, sleep regulation, sleep quality, diagnosis, and treatment need to be addressed. Identifying the underlying nature of sex and gender differences in sleep research has potential to accelerate improved care for both men and women facilitating better diagnosis, treatment, and ultimately prevention of sleep disorders and related comorbid conditions.
\end{abstract}

\section{Introduction}

$\mathbf{S}_{\mathrm{s}}^{\mathrm{E}}$ X AND GENDER DIFFERENCES cause men and women to sleep differently and may underlie the differential risk for sleep disorders. ${ }^{1}$ Sex differences refer to biological and physiological differences between men and women, with the sex chromosomes and the gonadal hormones primarily contributing to these differences at the cellular, organ, and system levels. A combination of environmental, social, and cultural influences on the biological factors in men and women contribute to gender differences.Table 1 lists a few examples of sex and gender differences in normal sleep and sleep disorders.

Distinct hormonal and physical changes at specific time points, such as puberty, pregnancy, and menopause, during a woman's lifespan can impact her sleep health and lead to gender-specific clinical disorders. Sleep disorders such as the restless legs syndrome (RLS), obstructive sleep apnea (OSA) and insomnia are more prevalent in women during these specific time points. ${ }^{24}$ Lack of adequate sleep or the presence of sleep disorders can greatly impact a woman's daily life, including her societal roles in the work force and as the primary caregiver in the family. ${ }^{24}$

The first major symposium dedicated to the topic of women and sleep was organized by the National Sleep Foundation in 2007 in Washington, DC. A series of articles detailing highlights of this symposium were published in 2008 in this journal. ${ }^{24-27}$ Since then, other symposia have addressed sex differences in sleep ${ }^{28}$ or women's sleep health, but significant knowledge gaps in research and lack of awareness of sleep issues relevant to women still exist.

Sex differences in research findings have important clinical consequences. For instance, last year, the Food and Drug Administration (FDA) reduced the recommended dose of zolpidem (Ambien ${ }^{\mathrm{TM}}$ ) for women by half. ${ }^{29}$ Zolpidem is a sedative-hypnotic benzodiazepine receptor agonist (BZRA) prescribed for insomnia treatment. This change in dosing was based on the discovery that women metabolized the same

The Society for Women's Health Research (SWHR), Washington, DC. 
Table 1. Examples of Sex and Gender Differences in Sleep

A. Epidemiology of normal sleep in general population

1. Sleep latency is longer in women than men; ${ }^{2}$

2 . Women $<55$ years report more sleepiness than men; ${ }^{2}$

3. Older women report 20 minutes less sleep than men; ${ }^{2}$

4. Women have more (106\%) SWS and less NREM stage 1 sleep than men; ${ }^{3}$

5. Men have more NREM stage 1 and stage 2 sleep than women; ${ }^{3}$

6. Normalized delta activity in older women is lower than in older men. ${ }^{4}$

B. Normal sleep in animal models

1. Female mice spend more time awake and less time in NREM than male mice, ${ }^{5}$

2. Female rats show $\sim 50 \%$ decrease in REM sleep compared with male rats; ${ }^{6}$

3. Slow wave activity during recovery dissipates more quickly in gonadectomized male rats compared female rats; ${ }^{5}$

4. Restraint stress produces increase in REM sleep which is greater in male mice than females; ${ }^{7}$

5. Sex-steroid modulation is greater in females than in male rats; ${ }^{6}$

6. Young male fruit flies have bimodal (middle of the day and night) sleep and young female flies sleep mostly at night. ${ }^{8}$

C. Epidemiology of sleep disorders

1. Women are at $40 \%$ increased risk for developing insomnia compared with men; ${ }^{9}$

2. Women are at twice the risk for RLS compared with men; ${ }^{10}$

3. Women with RLS are at higher risk for comorbid problems compared with men; ${ }_{12}^{11}$

4. Antidepressant use is more strongly associated with RLS in men than in women; ${ }^{12}$

5. Men are at twice the risk for OSA than women; $;^{13}$

6. REM sleep disordered breathing is more prevalent in women and men $\left(<55\right.$ years) ${ }^{14}$

7. Depression is more strongly associated with apnea in women (OR 5.2) than in men (OR 3.4). ${ }^{15}$

D. OSA symptoms and presentation

1. Women report different OSA symptoms than men; ${ }^{16,17}$

2. Men consistently have higher apnea-hypopnea index compared to women across all ages; ${ }^{18}$

3 . Waist-to-hip ratio is more predictive of severity of OSA in men than in women; ${ }^{19}$

4. Women have more partial obstructions compared with men, ${ }^{20}$

5. Women have lower scores than men on Epworth Sleepiness Scale, which maybe be more sensitive to subjective sleepiness in men than in women; ${ }^{17}$

6. Central nervous system white matter changes are more likely to occur in women with OSA than men. ${ }^{21}$

\section{E. Treatment}

1. Women may require less CPAP pressure for OSA treatment of similar severity in men; ${ }^{22}$

2. Women metabolize zolpidem $50 \%$ slower than men. ${ }^{23}$

Sex and gender differences exist in normal sleep and sleep disorders. Some examples discussed during the roundtable are listed.

CPAP, continuous positive airway pressure; NREM, non-rapid eye movement; OR, odds ratio; OSA, obstructive sleep apnea; REM, rapid eye movement; RLS, restless legs syndrome; SWS, slow wave sleep.

dose of zolpidem slower than men resulting in 50\% higher serum levels. ${ }^{23}$ This is the first time that the FDA has issued a sex-specific guideline for any drugs and this historical move underscores the need, once again, to fully understand the importance of sex differences in sleep disorders and treatments.

To address the current state of women's sleep health and determine the role of sex differences in sleep research, the Society for Women's Health Research (SWHR) convened an interdisciplinary panel of sleep experts (Table 2) for a roundtable discussion on October 24-25, 2013, in Washington, DC. The objectives of this meeting were to (1) review sex and gender differences in various areas of sleep health; (2) identify key knowledge gaps related to sex and gender differences in sleep; and (3) develop research recommendations to address these gaps. The participants were assigned to one of the four topic areas: (1) sex and gender differences in clinical research; (2) biological basis for sex and gender differences in sleep; (3) sleep related challenges specific to women; and (4) effective sleep therapies in women. Prior to the roundtable, SWHR charged the participants to collaboratively examine sex and gender differences in sleep and identify knowledge gaps in their assigned subtopic. A representative from each subgroup was chosen by the participants to summarize the subgroup's collective thoughts at the meeting. The discussion highlights from the roundtable are described below.

\section{Sex and Gender Differences in Clinical Research}

\section{Epidemiology of sex differences in normal sleep and sleep disorders}

Sex differences exist in sleep quality, duration, latency, and architecture in the general population. ${ }^{2}$ Sleep latency is defined as the number of minutes it takes to fall asleep and is longer in women compared with men (M.M. Ohayon personal communication). Further, discordance exists between subjective versus objective measures of sleep quality between men and women; women complain of poorer sleep quality and yet the quantitative analysis of their polysomnographic sleep does not support this claim. ${ }^{30}$ Additionally, women have increased slow wave sleep (SWS) as compared with men at any given age, and SWS decreases with age in men but not in women. ${ }^{3}$ Since these sex differences in SWS can be easily captured by electroencephalography, the possibility of using SWS as a biomarker for aging was suggested.

There are also sex differences in the prevalence of sleep disorders. Narcolepsy may have a slight male predominance, 
Table 2. Society for Women's Health Research Sleep Roundtable Participants List

A. Sex and gender differences in clinical research

1. Susan Redline, MD, MPH, Professor, Sleep Medicine, Harvard Medical School

2. Hrayr Attarian, MD, Associate Professor, Department of Neurology, Northwestern University

3. Helene Emsellem, MD, Medical Director, The Center for Sleep and Wake Disorders

4. Maurice M. Ohayon, MD, DSc., PhD, Professor, Department of Psychiatry and Behavioral Science, Stanford University

B. Biological basis for sex and gender differences in sleep

1. Jessica Mong, PhD, Associate Professor, Department of Pharmacology, University of Maryland

2. Fiona Baker, PhD, Senior Program Director, Center for Health Sciences, SRI International

3. Meir Kryger, MD, FRCPC, Professor, Department of Pulmonary Medicine, Yale University

4. Ketema Paul, PhD, Associate Professor, Department of Neurobiology, Morehouse School of Medicine

C. Sleep-related challenges specific to women

1. Joyce A. Walsleben, RN, PhD, Associate Professor, New York University School of Medicine

2. Sonia Ancoli-Israel, PhD, Professor, Department of Psychiatry, University of California at San Diego

3. Kathryn Lee, RN, CBSM, PhD, FAAN, Professor and Associate Dean for Research, University of California at San Francisco

D. Effective sleep therapies in women

1. Andrew Krystal, MD, MS, Professor, Department of Psychiatry, Duke University

2. Helene Emsellem, MD, Medical Director, The Center for Sleep and Wake Disorders

3. Michael T. Smith, PhD, CBSM, Professor, Department of Psychiatry and Behavioral Science, Johns Hopkins Bayview Medical Center

4. Thomas Roth, PhD, Professor, Department of Psychiatry, University of Michigan

Participants were assigned to either one or two topic areas and were charged to examine sex and gender differences in their assigned topic area. One participant (name highlighted in bold) was chosen by each subgroup as the group leader.

as does rapid eye movement (REM) behavior disorder (RBD), while idiopathic hypersomnia has a more of female preponderance (1.8/1); however, there is very little epidemiological data available for these disorders. ${ }^{1,31,32,33}$ Although women are at twice the risk for RLS as compared with men, the sex differences are a result of parity. While nulliparous women have prevalence similar to men, the risk for RLS gradually increases with number of pregnancies. Women with three or more children have three times greater risk of RLS compared with men or nulliparous women. ${ }^{10}$ This risk increases two-fold from pregnancy to menopause. ${ }^{10}$ Importantly, RLS follows a chronic course and may worsen with age. ${ }^{34}$ Women with RLS are at an increased risk of comorbid problems and are slightly more likely to complain of sleep related symptoms compared with men. ${ }^{11}$

The insomnia risk emerges with the onset of menses contributing to the gender differences. ${ }^{35}$ Although hormonal changes are implicated, the exact biological mechanism for increased risk of insomnia in girls is unknown. ${ }^{28,36}$ Interestingly, the two-fold increase in depression risk coincides with insomnia at puberty. ${ }^{37}$ For OSA, large gender differences in the prevalence have been attributed in part to pathophysiologic differences and in part to referral bias. ${ }^{13,38}$ Interestingly, weight gain in women versus obesity in men below the age of 50 years, was the cause of increased OSA prevalence. ${ }^{39,40}$

\section{Sex and gender differences in OSA symptoms and presentation}

Gender differences exist in how men and women report symptoms of OSA; men frequently report snoring, snorting, gasping and sleepiness, while women report unrefreshing sleep, fatigue, insomnia, and depression. ${ }^{16-15}$ Possible reasons for these differences may include less astute bed partners and that women are less likely to report snoring or snorting due to social awkwardness. Anatomical differences of the upper airway may further contribute to the underlying symptoms. The presence of more airway fat, greater neck circumference, and susceptibility of the airway to collapse is greater in men as compared with women. ${ }^{19}$ Regardless, the use of the right instruments in the right populations is crucial for a correct diagnosis of OSA in women. For example, an older screening instrument such as the STOPBang was designed with questions biased towards men, therefore making it inappropriate for use in women. Lack of gender-specific instruments is a major gap.

\section{Gender bias in sleep clinics}

The male:female (M:F) ratio of OSA is 2:1 in the community versus $8: 1$ in sleep centers. ${ }^{38}$ This discrepancy suggests that women are being understudied and undertreated for OSA. Similar gender biases in referral patterns exist for other sleep disorders, such as the RBD and insomnia. The M:F ratio for RBD in the community is approximately $2: 1^{31}$ versus 6:1 in the clinic $(\mathrm{H}$. Attarian, personal communication). In contrast, $\mathrm{M}: \mathrm{F}$ ratio for insomnia complaints is $1: 2^{41}$ versus approximately 1:5 in sleep clinics (H. Attarian, personal communication). Underrecognition of these sleep disorders, misdiagnosis and inappropriate treatment may further exacerbate these gender differences in clinical populations. Interestingly, a gender bias was observed in the diagnosis and treatment of OSA in sleep clinics, where women were younger, significantly heavier than males, and underwent delayed treatment for OSA. Women under the age of 55 years had to have a higher mean body mass index (BMI) of $41 \mathrm{~kg} /$ $\mathrm{m}^{2}$ before being treated for OSA as compared to men (mean BMI of $\left.35 \mathrm{~kg} / \mathrm{m}^{2}\right) .{ }^{38}$ It is possible that women represent their symptoms differently than men, contributing to the gender 
bias in misdiagnosis of OSA. Lack of awareness among physicians about OSA in women and perhaps misinterpretation of a woman's symptoms could also be the cause for misdiagnosis and mistreatment. ${ }^{42}$ For example, women with OSA were three times more likely to be treated for depression, three times likely to be on antidepressants potentially leading to weight gain, and much more likely to present with clinical insomnia. ${ }^{43}$

In summary, the underlying causes of gender differences in in some aspects of normal sleep and sleep disorders are unknown. Strong epidemiological associations have been found between sleep apnea and insomnia with comorbid depression and pain, but the biological underpinnings of these associations are unknown. Further, the use of gender- and ageappropriate tools to screen and diagnose sleep disorders by clinicians is essential. Finally, physicians need to be aware that sex differences exist in the presentation and underlying physiology of certain sleep disorders for them to correctly diagnose sleep disorders in women.

\section{Biological Basis for Sex and Gender Differences in Sleep}

\section{Usefulness of animal models in sleep}

Basic animal models have been critical for investigating mechanisms of sex differences. They have been useful in studying the effects of reproductive hormones directly on sleep behavior, sleep genetics, and the neurochemistry of the central nervous system. Rodent models of sleep are well established, and over the years, several published studies have used these models to successfully establish the role of sex steroids in contributing to sex differences in sleep regulation. ${ }^{6,44-47}$ Mouse models were also used to a lesser extent to determine the role of sex steroids in sleep; however, these models have primarily been useful in determining the role of genetic factors in causing sex differences in sleep regulation. ${ }^{5,48}$ Fruit flies have been used to investigate sex differences in sleep traits and make an excellent model to study sex differences in the influence of aging processes on sleep. ${ }^{49,8}$ For example, young female flies sleep mostly at night and exhibit increased daytime sleep and decreased nighttime sleep with increasing age. Interestingly, the male flies have a different sleep pattern compared to female flies. ${ }^{8}$

A major drawback to the existing animal models is the difference in the sleep patterns of these animals compared with humans. Humans or nonhuman primates have a consolidated sleep during the dark phase. In contrast, sleep in rodents is polyphasic (sleep in short cycles) during the light phase, thereby making them imperfect for studying human sleep behavior. Two gaps were highlighted with regard to animal models: (1) a lack of appropriate models to study human sleep behavior, and (2) a lack of appropriate animal models that can mimic human sleep disorders.

\section{Sex hormones and sex chromosome modulation of sleep}

Little evidence exists regarding the origin of sex differences in sleep behavior; however, studies in rats and mice have established a direct effect of hormones in causing these differences. Female mice are more awake in the dark phase compared with the male mice, and gonadectomy eliminates these sex differences. ${ }^{5}$ Similarly, gonadectomy in female and male rats eliminates all sex differences in the sleep-wake cycle and adding back physiological levels of sex-specific steroids restores these differences. ${ }^{6}$ Importantly, ovarian steroids suppress non-rapid eye movement (NREM) and REM sleep with a corresponding increase in waketime in these female rats. Further, the suppression is greater in female rats compared to the male rats. ${ }^{6}$ Interestingly, both increases in estradiol and progesterone levels during the late luteal phase in women correlate with an increase in wakefulness and a decrease in REM sleep, thereby coinciding with the observations seen in female rats. ${ }^{50}$ Finally, new emerging evidence suggests that estradiol consolidates circadian sleepwake rhythms in female rats lending to its novel role in interacting with both sleep and circadian regulation. ${ }^{51}$ Evidence for the role of sex chromosomes in sleep regulation comes from two observations in mice: (1) sex differences in sleep propensity are not entirely eliminated by gonadectomy ${ }^{5}$ (e.g., dissipation of SWA during recovery occurs more quickly in gonadectomized males compared with gonadectomized female mice); and (2) dissipation of sleep pressure after sleep loss is partially dependent on sex chromosomes. ${ }^{48}$ Eventually, sex hormones and genetic mechanisms in conjunction with cultural and societal demands are thought to drive the sex and gender differences in sleep and sleep pathologies in humans.

\section{Biological mechanisms underlying sex differences in sleep}

Sex hormones organize the sleep-wake patterns during early development by acting on the ventrolateral preoptic area, an established sleep promoting nucleus in the brain. ${ }^{6}$ Interestingly, the ventrolateral preoptic area in female rats, but not male rats, is sensitive to changes in sex hormones. ${ }^{52,6}$ Another organized circuitry in the brain, called the orexin/ hypocretin system, is modulated by gonadal hormones and is thought to have an important role in the regulation of sleep and arousal states. ${ }^{53,54}$ The hormonal modulation of the orexin/hypocretin system is greater in females than in male rats. ${ }^{53}$ Current studies have now begun to focus on the mechanisms underlying these sex differences and identifying neurochemical circuits involved in regulating sleep and wake patterns that are sensitive to the effects of estradiol. However, the exact role of estrogen in modulating sleep patterns in the brain is still unknown.

Novel emerging technologies such as optogenetics, Designer Receptor Exclusively Activated by Designer Drugs, and forward genetics are now being used by researchers to target and manipulate the arousal systems in animal models to further investigate mechanisms of sex differences in normal sleep and sleep disorders. These novel techniques combined with further research in animal models may allow several investigations into unanswered basic research needs.

In summary, despite the advances made in basic science in sleep mechanisms and regulation, many knowledge gaps persist regarding sex differences. In part, these gaps have been difficult to address due to the lack of appropriate animal models that can mimic human sleep behavior accurately. Besides the need for animal models for chronic insomnia and sleep apnea, these models should mimic the striking sex differences seen in humans in order to understand the 
biological mechanisms that lead to these sex differences. Although major advances have been made in basic research of sex differences, there is a lack of synergy with clinical findings. For the most part, basic and clinical research on sex differences has been done in silos. It is imperative that basic research, in the future, address relevant questions with clinical implications.

\section{Sleep-Related Challenges Specific to Women}

\section{Impact of hormonal transitions on sleep}

Normal sleep in women is impacted by hormonal effects during menses, pregnancy/lactation, perimenopause, menopause, and post menopause and often leads to sleep disturbances or sleep disorders during these periods. For example, one-third of women complain of sleep disturbances and related symptoms such as cramps, bloating, and headaches as reasons for disrupted sleep during the premenstrual week or during menses. ${ }^{55}$ Menopause is characterized by cessation of menses, associated with an increase in follicle stimulating hormone and a decrease in estrogen levels. The prevalence of insomnia is increased from $33 \%-36 \%$ in premenopausal women to $44 \%-61 \%$ in postmenopausal women. ${ }^{1}$ This increase may be associated with the presence of vasomotor symptoms, hormonal changes, age-associated changes in sleep, comorbid conditions, and psychosocial factors. ${ }^{56}$ Hormone replacement therapy (HRT) seems to alleviate some of the sleep disturbances and improve quality of life; however, the role of HRT in improving sleep quality has been debatable and still continuous to be a major research gap, largely due to lack of concurrence between the study populations and study variables. $^{57-60}$ Recent longitudinal studies have begun to address major questions about the link between menopause and poor sleep in racially diverse populations. For example, Study of Women's Health Across the Nation (SWAN) and the Penn Ovarian Study have incorporated sleep-related items into their questionnaires and performed polysomnographic studies on subgroups. ${ }^{61,62}$ These studies have made major advances in identifying underlying causes of sleep disturbance in menopause and have begun to explore whether there are differences associated with ethnicity and menopausal status.

\section{Psychosocial factors related to poor sleep quality}

Psychosocial issues impact sleep health in women much more than men. The prevalence of depression is higher in women starting with puberty and is linked to insomnia. ${ }^{35}$ More midlife women (66\%) than men are caregivers and are more likely to report stress, depression, and sleep disturbances. ${ }^{63,64}$ Further, women constitute half the workforce, and women shift workers have greater difficulty adjusting to shift work, in part due to sleep problems. ${ }^{65}$ Women shift workers report poor sleep quality, an increased risk of breast cancer, shorter menstrual cycles, and a greater risk for miscarriage, menstrual disruption, and subfertility. ${ }^{65,66}$ There is a need to understand how to integrate these unique psychosocial issues into biological research. It is also essential to understand what quality of life indicators matter to women in their midlife and what causes stress in women of different cultures. Finally, women veterans are an important population in which sleep problems have received scant attention; posttraumatic stress disorder is a major risk factor for in- somnia in general and is related to more severe sleep disruption in women veterans. ${ }^{67}$

\section{Physical factors and poor sleep quality}

Conditions besides sleep disorders-such as overactive bladder $(\mathrm{OAB})$ and pain-can cause disrupted sleep in women. Fibromyalgia, a chronic pain condition, affects $7 \%$ of women over the age of 60 years and is characterized by poor sleep efficiency. ${ }^{68}$ Disrupted sleep is associated with poor daytime function, decline in physical performance, and functional limitations in older women. ${ }^{69}$ As nighttime sleep deviates from 7 hours, older women experienced difficulty with daily activities leading to poor quality of life. ${ }^{69}$ However, more longitudinal studies are needed to determine if poor sleep is associated with subsequent decline in physical function.

In summary, possibly the lack of appropriate variables and methods to measure sleep quality in women may underlie the discrepancy between the objective measures and subjective reports. Designing better longitudinal studies with appropriate variables and populations may help address some of the challenges faced by women regarding their sleep health. Importantly, enhancing the distribution of these research findings to health care professionals is essential to making progress in treating women with sleep-related conditions and disorders.

\section{Effective Sleep Therapies for Women}

\section{General treatment considerations}

General treatment of sleep disorders should be different for women as compared to men. Prior to administering treatment in women, physicians must be aware of the following: (1) risk for adverse pregnancy outcomes for women of child-bearing age; (2) risk associated with adverse effects of drugs (excreted through breast milk) on nursing infants; (3) possible ineffectiveness of birth-control pills by ingestion of other medications (e.g., modafinil and R-modafinil) due to increased metabolism of birth control pills; and 4) prescribing lower doses of some drugs, since women tend to clear these drugs less effectively than men (e.g., all zolpidem products). As discussed above, zolpidem is the only hypnotic that has shown gender-specific changes in metabolism. ${ }^{23,70}$

\section{Therapies administered during hormonal transitions in women}

Peri- and post-menopausal sleep disturbance. Sleep disturbance, particularly nocturnal awakenings, is common in peri-/post-menopausal women, and importantly, the most suitable therapy in these women has been treatment of sleepmaintenance insomnia. The BZRAs, zolpidem and eszopiclone, have been effective than placebo in the treatment of insomnia in these women; however, only three trials were conducted using the BZRAs. ${ }^{71-73}$ Zolpidem (10mg) was effective in maintaining sleep in these women, ${ }^{71}$ but the dosage used in this study was twice the FDA recommended dose for women. Similarly, eszopiclone $(3 \mathrm{mg}$ ) improved sleeponset, sleep-maintenance insomnia, and associated comorbid symptoms in peri-/post-menopausal women. ${ }^{72,73}$ HRT has been shown to improve sleep in some post-menopausal women with insomnia; however, the results are inconclusive. ${ }^{74-76}$ Further, the associated risk suggested by the Women's health 
initiative data has affected the administration of HRT for these women. ${ }^{77}$ Cognitive behavioral therapy (CBT) may be an effective treatment for peri-/post-menopausal insomnia, based on the rationale that insomnia in these women is due to behavioral conditioning. CBT may be beneficial in conjunction with other therapies or when insomnia fails to respond to HRT or occurs without night sweats. ${ }^{78}$ Early on, night sweats may precipitate insomnia in premenopausal women, but over time other perpetuating factors lead to chronic insomnia with certain behavioral components. Although CBT in peri-/post-menopausal women may be effective, efficacy studies with CBT in these women are only beginning to be conducted.

Pre-menstrual sleep disturbances. Premenstrual sleep disturbance may occur with mood symptoms in the late luteal phase of the menstrual cycle. ${ }^{79,80}$ Poor sleep quality may be associated with high anxiety levels in women with severe premenstrual syndrome. Interestingly, severe mood-related premenstrual symptoms may be a result of serotonin deficiency. ${ }^{50}$ In such cases, a short course over several days to a week of serotonin reuptake inhibitors (SSRIs) administered during the late luteal phase of the menstrual cycle has been shown to be effective in treating the mood symptoms; however, data on the efficacy of SSRIs in treating premenstrual sleep disturbance is lacking. ${ }^{81,80}$ A short course of hypnotics or proactive use of CBT may also be effective in treating isolated premenstrual insomnia; once again, there is lack of data regarding these practices.

Pregnancy and post-partum related sleep disturbances. Sleep disturbance, particularly during the third trimester of pregnancy, can be due to many factors such as discomfort, urinary frequency, fetal activity, RLS, or OSA. Careful managed care is essential in women who get pregnant or plan to get pregnant when they are on medications for narcolepsy, idiopathic hypersomnolence, and insomnia. A careful riskbenefit analysis is essential and sometimes may necessitate discontinuation of medications in these women. Currently, drugs used for treating excessive sleepiness (amphetamines, methylphenidate, modafinil), insomnia (zolpidem, zaleplon, eszopiclone, ramelteon, doxepin), and RLS (pramipexole, ropinirole, rotigotine, and gabapentin) are designated as class $\mathrm{C}$ drugs. According to the FDA pregnancy categories, class $\mathrm{C}$ designation means that "animal reproduction studies have shown an adverse effect on the fetus and there are no adequate and well controlled studies in humans, but potential benefits may warrant use in pregnant women despite potential risks." This statement also suggests that there are no human studies with any of these drugs and the available data comes from surveillance data collected on pregnant women who had no choice but to remain on the treatment. Treating new onset

Table 3. Proposed Research Recommendations in Sleep Research from the Society for Women's Health Research Sleep Roundtable

A. Examine sex differences to

1. Understand hormonal effects on sleep mechanisms;

2. Determine the risk for sleep disorders;

3. Study circadian rhythms disruption leading to sleep disorders;

4. Determine stress and pain response to sleep deprivation;

5. Understand the effect of sedatives on sleep mechanisms;

6. Analyze pharmacodynamics of existing sleep drugs;

7. Determine fetal outcomes to poor sleep quality; and

8. Analyze large available datasets for novel biomarkers, objective sleep quality measures, and genetic polymorphisms.

B. Examine gender differences to

1. Explore response to novel drugs;

2. Understand source of objective and subjective discordance;

3. Determine CPAP adherence;

4. Understand social determinants for diagnosis of sleep disorders; and

5. Analyze sleep patterns of various ethnic, racial, and socioeconomic groups.

C. Utilize animal models to

1. Study steroid mechanism on neural circuitry and neurochemistry;

2. Understand sleep and circadian disruption on reproductive function;

3. Determine relationship between sleep disturbances and inflammation;

4. Understand the effect of sex hormones on sedative-hypnotics; and

5. Determine the link between sex-specific sleep mechanisms and PTSD.

D. Conduct studies specifically in women to

1. Understand the relationship between comorbid insomnia and sleep apnea;

2. Utilize best combination therapies for maximal results;

3. Compare different treatment modalities for menopausal sleep disturbances;

4. Determine effective management of new onset sleep disorders during pregnancy;

5. Determine effective therapies for premenstrual sleep disturbances;

6. Understand change in drug response as a function of menstrual cycle and menopausal status;

7. Develop screening instruments for sleep patterns pertinent to women; and

8. Develop novel techniques to reconcile objective and subjective reporting.

Based on existing knowledge gaps in the sleep field, the discussions at the roundtable meeting led to the identification of key recommendations listed in the table.

PTSD, posttraumatic stress disorder. 
sleep disturbances in pregnant women is a challenge and more research is needed on management of these disturbances since there are no treatment studies to guide physicians. Many physicians have advocated proactive use of continuous positive airway pressure (CPAP) for OSA and behavioral interventions for managing sleep disturbances during pregnancy.

Similar treatment concerns also exist for postpartum women with sleep disorders. ${ }^{82,83}$ Excretion of drugs into breast milk is a concern; therefore, women are recommended to breastfeed when the drug level in the blood is as low as possible However, it is not known whether all drugs are excreted in breast milk and some stimulant drugs such as amphetamines and methylphenidate are contraindicated. Further, dopaminergic drugs used for RLS, in fact, block prolactin release, affecting milk production. New onset sleep disturbance can arise from postpartum depression, which may be a result of a baby's inability to sleep through the night and sometimes due to development of insomnia. ${ }^{82,84}$ Therefore, due to the lack of controlled trials during lactation, a complex risk/benefit analysis of breast feeding and need for the drug is required by the physicians. ${ }^{82}$ If the drug is absolutely needed, patients are advised to avoid breastfeeding near peak blood levels. Importantly, physicians must provide effective therapy for their patients that will prevent decreased responsiveness and infant caretaking capacity regardless of the mother breastfeeding her child. Lastly, it is important and critical to distinguish insomnia in postpartum women from regular sleep disturbances so that effective and preferential treatment such as CBT may be used. Depending on the breastfeeding status, judicious use of medications with the fewest associated risks may be used if CBT is ineffective.

\section{Management of sleep disorders and comorbidities}

Women require less CPAP pressure for treatment of similar severity of OSA as compared with men; however, data on CPAP adherence in women is unavailable. Women have twice the risk for depression compared with men, and the increased risk is particularly prevalent during the period of menarche to menopause. ${ }^{85,86}$ Depression accounts for roughly $23 \%$ of all insomnia, although it is not necessarily cause and effect. ${ }^{87,88}$ SSRIs have been used to treat depression in women, and women preferentially respond to these drugs compared with men. ${ }^{89,90}$ Importantly, sleep in these women may improve or be disturbed by SSRIs, and as a result adjunctive insomnia therapy is generally recommended. ${ }^{84} \mathrm{OAB}$ is a bladder storage problem associated with incontinence and nocturia, and women exhibit more severe symptoms than men. ${ }^{91}$ Diagnosis for $\mathrm{OAB}$ can be confused with insomnia since $\mathrm{OAB}$ syndrome patients generally have frequent awakenings. Over time, OAB can lead to chronic insomnia. Since the treatments for insomnia and $\mathrm{OAB}$ are different, a correct diagnosis to differentiate between insomnia and $\mathrm{OAB}$ requires a bladder diary combined with a sleep diary.

In summary, a careful risk-benefit analysis is important to consider for BZRA dosage, especially in view of the recent FDA mandate, particularly in women who have been successfully and effectively treated with zolpidem for an extended period of time. Lack of systematic studies for most sleep therapies in women is a major gap. As an example, the
Asthma Clinical Research Network model could be applied in learning about the side effects, gender differences and comorbidities of sleep therapies. ${ }^{92}$ Finally, it is most essential to recapitulate these clinical studies in basic science models or vice versa.

\section{Research Recommendations and Conclusion}

Discussions at the roundtable resulted in several research recommendations (Table 3). An established program such as the SWHR's interdisciplinary networks may be the platform needed to address some of these identified research needs in women's sleep health. Ultimately, understanding sex differences in sleep and sleep disorders will allow for better diagnosis, treatment, and eventually prevention of these disorders in women.

\section{Acknowledgments}

SWHR would like to acknowledge Jazz Pharmaceuticals for their generous support. A special thanks to SWHR staff members Dr. Dario Dieguez for technical support and Alana Lerer for logistical support.

\section{Author Disclosure Statement}

No competing financial interests exist.

\section{References}

1. Krishnan V, Collop NA. Gender differences in sleep disorders. Curr Opin Pulm Med 2006;12:383-389.

2. Ohayon MM, Reynolds CF, 3rd, Dauvilliers Y. Excessive sleep duration and quality of life. Ann Neurol 2013;73: 785-794.

3. Redline S, Kirchner HL, Quan SF, Gottlieb DJ, Kapur V, Newman A. The effects of age, sex, ethnicity, and sleepdisordered breathing on sleep architecture. Arch Intern Med 2004; 164:406-418.

4. Latta F, Leproult R, Tasali E, Hofmann E, Van Cauter E. Sex differences in delta and alpha EEG activities in healthy older adults. Sleep 2005;28:1525-1534.

5. Paul KN, Dugovic C, Turek FW, Laposky AD. Diurnal sex differences in the sleep-wake cycle of mice are dependent on gonadal function. Sleep 2006;29:1211-1223.

6. Cusmano DM, Hadjimarkou MM, Mong JA. Gonadal steroid modulation of sleep and wakefulness in male and female rats is sexually differentiated and neonatally organized by steroid exposure. Endocrinology 2014;155: 204-214.

7. Paul KN, Losee-Olson S, Pinckney L, Turek FW. The ability of stress to alter sleep in mice is sensitive to reproductive hormones. Brain Res 2009;1305:74-85.

8. Koh K, Evans JM, Hendricks JC, Sehgal A. A Drosophila model for age-associated changes in sleep:wake cycles. Proc Natl Acad Sci U S A 2006;103:13843-13847.

9. Zhang B, Wing YK. Sex differences in insomnia: A metaanalysis. Sleep 2006;29:85-93.

10. Berger K, Luedemann J, Trenkwalder C, John U, Kessler C. Sex and the risk of restless legs syndrome in the general population. Arch Intern Med 2004;164:196-202.

11. Bentley AJ, Rosman KD, Mitchell D. Gender differences in the presentation of subjects with restless legs syndrome. Sleep Med 2006;7:37-41. 
12. Baughman KR, Bourguet CC, Ober SK. Gender differences in the association between antidepressant use and restless legs syndrome. Mov Disord 2009;24:1054-1059.

13. Jordan AS, McEvoy RD. Gender differences in sleep apnea: Epidemiology, clinical presentation and pathogenic mechanisms. Sleep Med Rev 2003;7:377-389.

14. Koo BB, Dostal J, Ioachimescu O, Budur K. The effects of gender and age on REM-related sleep-disordered breathing. Sleep Breath 2008;12:259-264.

15. Wheaton AG, Perry GS, Chapman DP, Croft JB. Sleep disordered breathing and depression among U.S. adults: National Health and Nutrition Examination Survey, 20052008. Sleep 2012;35:461-467.

16. Kump K, Whalen C, Tishler PV, et al. Assessment of the validity and utility of a sleep-symptom questionnaire. Am J Respir Crit Care Med 1994;150:735-741.

17. Baldwin CM, Kapur VK, Holberg CJ, Rosen C, Nieto FJ, Sleep Heart Health Study G. Associations between gender and measures of daytime somnolence in the Sleep Heart Health Study. Sleep 2004;27:305-311.

18. Gabbay IE, Lavie P. Age- and gender-related characteristics of obstructive sleep apnea. Sleep Breath 2012;16:453-460.

19. Subramanian S, Jayaraman G, Majid H, Aguilar R, Surani $\mathrm{S}$. Influence of gender and anthropometric measures on severity of obstructive sleep apnea. Sleep Breath 2012;16: 1091-1095.

20. Anttalainen U, Saaresranta T, Kalleinen N, Aittokallio J, Vahlberg T, Polo O. CPAP adherence and partial upper airway obstruction during sleep. Sleep Breath 2007;11:171-176.

21. Macey PM, Kumar R, Yan-Go FL, Woo MA, Harper RM. Sex differences in white matter alterations accompanying obstructive sleep apnea. Sleep 2012;35:1603-1613.

22. Ralls FM, Grigg-Damberger M. Roles of gender, age, race/ ethnicity, and residential socioeconomics in obstructive sleep apnea syndromes. Curr Opin Pulm Med 2012;18: 568-573.

23. Cubala WJ, Wiglusz M, Burkiewicz A, Galuszko-Wegielnik M. Zolpidem pharmacokinetics and pharmacodynamics in metabolic interactions involving CYP3A: Sex as a differentiating factor. Eur J Clin Pharmacol 2010;66:955; author reply 957-958.

24. Lee KA, Kryger MH. Women and sleep. J Womens Health (Larchmt) 2008;17:1189-1190.

25. Paul KN, Turek FW, Kryger MH. Influence of sex on sleep regulatory mechanisms. J Womens Health (Larchmt) 2008; 17:1201-1208.

26. Lee KA, Baker FC, Newton KM, Ancoli-Israel S. The Influence of reproductive status and age on women's sleep. J Womens Health (Larchmt) 2008;17:1209-1214.

27. Phillips BA, Collop NA, Drake C, Consens F, Vgontzas AN, Weaver TE. Sleep disorders and medical conditions in women. Proceedings of the Women and Sleep Workshop, National Sleep Foundation, Washington, DC, March 5-6, 2007. J Womens Health (Larchmt) 2008;17:1191-1199.

28. Mong JA, Baker FC, Mahoney MM, et al. Sleep, rhythms, and the endocrine brain: influence of sex and gonadal hormones. J Neurosci 2011;31:16107-16116.

29. Food and Drug Administration (FDA). FDA requiring lower recommended dose for certain sleep drugs containing zolpidem: Reminder about risk of impaired activities the morning after use for all insomnia drugs. FDA News Release, Jan 10, 2013. Available at: www.fda.gov/newsevents/ newsroom/pressannouncements/ucm334798.htm Accessed March 6, 2014.
30. Baker FC, Kahan TL, Trinder J, Colrain IM. Sleep quality and the sleep electroencephalogram in women with severe premenstrual syndrome. Sleep 2007;30:1283-1291.

31. Bjornara KA, Dietrichs E, Toft M. REM sleep behavior disorder in Parkinson's disease-is there a gender difference? Parkinsonism Relat Disord 2013;19:120-122.

32. Ali M, Auger RR, Slocumb NL, Morgenthaler TI. Idiopathic hypersomnia: Clinical features and response to treatment. Journal of clinical sleep medicine. JCSM 2009; 5:562-568.

33. Vernet C, Arnulf I. Idiopathic hypersomnia with and without long sleep time: A controlled series of 75 patients. Sleep 2009;32:753-759.

34. Garcia-Borreguero D, Egatz R, Winkelmann J, Berger K. Epidemiology of restless legs syndrome: The current status. Sleep Med Rev 2006;10:153-167.

35. Johnson EO, Roth T, Schultz L, Breslau N. Epidemiology of DSM-IV insomnia in adolescence: Lifetime prevalence, chronicity, and an emergent gender difference. Pediatrics 2006;117:e247-256.

36. Knutson KL. The association between pubertal status and sleep duration and quality among a nationally representative sample of U.S. adolescents. Am J Hum Biol 2005; 17 : 418-424.

37. Born L, Shea A, Steiner M. The roots of depression in adolescent girls: Is menarche the key? Curr Psychiatry Rep 2002;4:449-460.

38. Redline S, Kump K, Tishler PV, Browner I, Ferrette V. Gender differences in sleep disordered breathing in a community-based sample. Am J Respir Crit Care Med 1994;149:722-726.

39. Young T, Peppard PE, Taheri S. Excess weight and sleep-disordered breathing. J Appl Physiol 2005;99: 1592-1599.

40. Redline S, Schluchter MD, Larkin EK, Tishler PV. Predictors of longitudinal change in sleep-disordered breathing in a nonclinic population. Sleep 2003;26:703-709.

41. Hale L, Do DP, Basurto-Davila R, et al. Does mental health history explain gender disparities in insomnia symptoms among young adults? Sleep Med 2009;10:1118-1123.

42. Young T, Hutton R, Finn L, Badr S, Palta M. The gender bias in sleep apnea diagnosis. Are women missed because they have different symptoms? Arch Intern Med 1996;156: 2445-2451.

43. Shepertycky MR, Banno K, Kryger MH. Differences between men and women in the clinical presentation of patients diagnosed with obstructive sleep apnea syndrome. Sleep 2005;28:309-314.

44. Branchey M, Branchey L, Nadler RD. Effects of estrogen and progesterone on sleep patterns of female rats. Physiol Behav 1971;6:743-746.

45. Zhang SQ, Kimura M, Inoue S. Sleep patterns in cyclic and pseudopregnant rats. Neurosci Lett 1995;193:125-128.

46. Fang J, Fishbein W. Sex differences in paradoxical sleep: influences of estrus cycle and ovariectomy. Brain Res 1996;734:275-285.

47. Schwierin B, Borbely AA, Tobler I. Sleep homeostasis in the female rat during the estrous cycle. Brain Res 1998; 811:96-104.

48. Ehlen JC, Hesse S, Pinckney L, Paul KN. Sex chromosomes regulate nighttime sleep propensity during recovery from sleep loss in mice. PloS One 2013;8:e62205.

49. Zimmerman JE, Chan MT, Jackson N, Maislin G, Pack AI. Genetic background has a major impact on differences in 
sleep resulting from environmental influences in Drosophila. Sleep 2012;35:545-557.

50. Baker FC, Sassoon SA, Kahan T, et al. Perceived poor sleep quality in the absence of polysomnographic sleep disturbance in women with severe premenstrual syndrome. J Sleep Res 2012;21:535-545.

51. Schwartz MD, Mong JA. Estradiol modulates recovery of REM sleep in a time-of-day-dependent manner. Am J Physiol Regul Integr Comp Physiol 2013;305:R271280.

52. Hadjimarkou MM, Benham R, Schwarz JM, Holder MK, Mong JA. Estradiol suppresses rapid eye movement sleep and activation of sleep-active neurons in the ventrolateral preoptic area. Eur J Neurosci 2008;27:1780-1792.

53. Silveyra P, Cataldi NI, Lux-Lantos V, Libertun C. Gonadal steroids modulated hypocretin/orexin type-1 receptor expression in a brain region, sex and daytime specific manner. Regul Pept 2009;158:121-126.

54. Ebrahim IO, Howard RS, Kopelman MD, Sharief MK, Williams AJ. The hypocretin/orexin system. J R Soc Med 2002;95:227-230.

55. National Sleep Foundation. 2007 Sleep in America poll. Washington, DC: National Sleep Foundation, 2007.

56. Joffe H, Massler A, Sharkey KM. Evaluation and management of sleep disturbance during the menopause transition. Semin Reprod Med 2010;28:404-421.

57. Antonijevic IA, Stalla GK, Steiger A. Modulation of the sleep electroencephalogram by estrogen replacement in postmenopausal women. Am J Obstet Gynecol 2000;182:277-282.

58. Heinrich AB, Wolf OT. Investigating the effects of estradiol or estradiol/progesterone treatment on mood, depressive symptoms, menopausal symptoms and subjective sleep quality in older healthy hysterectomized women: a questionnaire study. Neuropsychobiology 2005;52:17-23.

59. Kalleinen N, Polo O, Himanen SL, Joutsen A, Polo-Kantola P. The effect of estrogen plus progestin treatment on sleep: a randomized, placebo-controlled, double-blind trial in premenopausal and late postmenopausal women. Climacteric 2008;11:233-243.

60. Tranah GJ, Parimi N, Blackwell T, et al. Postmenopausal hormones and sleep quality in the elderly: a population based study. BMC Womens Health 2010;10:15.

61. Kravitz HM, Avery E, Sowers M, et al. Relationships between menopausal and mood symptoms and EEG sleep measures in a multi-ethnic sample of middle-aged women: The SWAN sleep study. Sleep 2011;34:1221-1232.

62. Sowers MF, Zheng H, Kravitz HM, et al. Sex steroid hormone profiles are related to sleep measures from polysomnography and the Pittsburgh Sleep Quality Index. Sleep 2008;31:1339-1349.

63. Family Care Giving Alliance fact sheet: Selected caregiver statistics. National Center for Caregiving, 2012. Available at http://www.caregiver.org/caregiver/jsp/print_friendly.jsp? nodeid=439 Accessed March 24, 2014.

64. Administration on Aging. Older Americans behavioral health issue brief 12: Caregivers as partners and clients of behavioral health services. Substance Abuse and Mental Health Services Administration, 2013. Available at http:// www.aoa.gov/AoARoot/AoA_Programs/HPW/Behavioral/ docs $2 /$ Issue\%20Brief\%2012\%20Caregivers.pdf Accessed March 24, 2014.

65. Chung SA, Wolf TK, Shapiro CM. Sleep and health consequences of shift work in women. J Womens Health (Larchmt) 2009;18:965-977.
66. Stocker L. Women working shifts are at greater risk of miscarriage, menstrual disruption and subfertility. ESHRE, 2013. Available at http://www.eshre2013.eu/ /media/Files/London/ Press\%20releases/stocker.pdf Accessed March 5, 2014.

67. Hughes J, Jouldjian S, Washington DL, Alessi CA, Martin JL. Insomnia and symptoms of post-traumatic stress disorder among women veterans. Behav Sleep Med 2013;11: 258-274.

68. Prados G, Miro E, Martinez MP, Sanchez AI, Lopez S, Saez G. Fibromyalgia: Gender differences and sleepdisordered breathing. Clin Exp Rheumatol 2013;31:S102-110.

69. Goldman SE, Stone KL, Ancoli-Israel S, et al. Poor sleep is associated with poorer physical performance and greater functional limitations in older women. Sleep 2007;30: 1317-1324.

70. Greenblatt DJ, Harmatz JS, von Moltke LL, et al. Comparative kinetics and response to the benzodiazepine agonists triazolam and zolpidem: Evaluation of sex-dependent differences. J Pharmacol Exp Ther 2000;293:435-443.

71. Dorsey CM, Lee KA, Scharf MB. Effect of zolpidem on sleep in women with perimenopausal and postmenopausal insomnia: a 4-week, randomized, multicenter, double-blind, placebo-controlled study. Clin Ther 2004;26:1578-1586.

72. Soares CN, Joffe H, Rubens R, Caron J, Roth T, Cohen L. Eszopiclone in patients with insomnia during perimenopause and early postmenopause: A randomized controlled trial. Obstet Gynecol 2006;108:1402-1410.

73. Joffe H, Petrillo L, Viguera A, et al. Eszopiclone improves insomnia and depressive and anxious symptoms in perimenopausal and postmenopausal women with hot flashes: A randomized, double-blinded, placebo-controlled crossover trial. Am J Obstet Gynecol 2010;202:171.e1-171.e11.

74. Pickett CK, Regensteiner JG, Woodard WD, Hagerman DD, Weil JV, Moore LG. Progestin and estrogen reduce sleep-disordered breathing in postmenopausal women. J Applied Physiol 1989;66:1656-1661.

75. Scharf MB, McDannold MD, Stover R, Zaretsky N, Berkowitz DV. Effects of estrogen replacement therapy on rates of cyclic alternating patterns and hot-flush events during sleep in postmenopausal women: a pilot study. Clin Ther 1997;19:304-311.

76. Polo-Kantola P, Erkkola R, Irjala K, Pullinen S, Virtanen I, Polo O. Effect of short-term transdermal estrogen replacement therapy on sleep: A randomized, double-blind crossover trial in postmenopausal women. Fertil Steril 1999;71:873-880.

77. Rossouw JE, Anderson GL, Prentice RL, et al. Risks and benefits of estrogen plus progestin in healthy postmenopausal women: principal results From the Women's Health Initiative randomized controlled trial. JAMA 2002;288:321-333.

78. Krystal AD, Edinger J, Wohlgemuth W, Marsh GR. Sleep in peri-menopausal and post-menopausal women. Sleep Med Rev 1998;2:243-253.

79. Leibenluft E, Fiero PL, Rubinow DR. Effects of the menstrual cycle on dependent variables in mood disorder research. Arch Gen Psychiatry 1994;51:761-781.

80. Eriksson E, Andersch B, Ho HP, Landen M, Sundblad C. Diagnosis and treatment of premenstrual dysphoria. J Clin Psychiatry 2002;63 Suppl 7:16-23.

81. Altshuler LL. The use of SSRIs in depressive disorders specific to women. Jf Clin Psychiatry 2002;63 Suppl 7:3-8.

82. Newport DJ, Hostetter A, Arnold A, Stowe ZN. The treatment of postpartum depression: minimizing infant exposures. J Clin Psychiatry 2002;63 Suppl 7:31-44. 
83. Moline ML, Broch L, Zak R, Gross V. Sleep in women across the life cycle from adulthood through menopause. Sleep Med Rev 2003;7:155-177.

84. Krystal AD. Depression and insomnia in women. Clin Cornerstone 2004;6:S19-S28.

85. Association AP. Women and depression. American Psychological Association. Available at http://www.apa.org/about/ gr/issues/women/depression.aspx Accessed March 5, 2014.

86. Burt VK, Stein K. Epidemiology of depression throughout the female life cycle. J Clin Psychiatry 2002;63 Suppl 7:9-15.

87. Ford DE, Kamerow DB. Epidemiologic study of sleep disturbances and psychiatric disorders. An opportunity for prevention? JAMA 1989;262:1479-1484.

88. Harris T. Depression in women and its sequelae. J Psychosom Res 2003;54:103-112.

89. Nishizawa S, Benkelfat C, Young SN, et al. Differences between males and females in rates of serotonin synthesis in human brain. Proc Natl Acad Sci U S A 1997;94: 5308-5313.

90. Ellenbogen MA, Young SN, Dean P, Palmour RM, Benkelfat $\mathrm{C}$. Mood response to acute tryptophan depletion in healthy volunteers: Sex differences and temporal stability. Neuropsychopharmacology 1996;15:465-474.

91. Stewart WF, Van Rooyen JB, Cundiff GW, et al. Prevalence and burden of overactive bladder in the United States. World J Urol 2003;20:327-336.

92. Szefler SJ, Chinchilli VM, Israel E, et al. Key observations from the NHLBI Asthma Clinical Research Network. Thorax 2012;67:450-455.

Address correspondence to: Christine L. Carter, PhD, MPH Vice President, Scientific Affairs Society for Women's Health Research 1025 Connecticut Ave, Northwest Suite 601 Washington, DC 20036

E-mail: chris@swhr.org 